\title{
Probing Magnetic Fields With SNRs
}

\author{
Roland Kothes \\ DRAO, NSI-NRCC, Penticton, Canada; email: roland.kothes@nrc-cnrc.gc.ca
}

\begin{abstract}
As supernova remnants (SNRs) expand, their shock waves freeze in and compress magnetic field lines they encounter; consequently we can use SNRs as magnifying glasses for interstellar magnetic fields. A simple model is used to derive polarization and rotation measure (RM) signatures of SNRs. This model is exploited to gain knowledge about the large-scale magnetic field in the Milky Way. Three examples are given which indicate a magnetic anomaly, an azimuthal large-scale magnetic field towards the anti-centre, and a chimney that releases magnetic energy from the plane into the halo.
\end{abstract}

Keywords. ISM: magnetic fields, supernova remnants

Introduction: Recently, there have been many studies of the Milky Way's large-scale magnetic field utilizing RM observations of compact polarized objects such as extragalactic point sources and pulsars. However, the magnetic field is averaged along the line of sight weighted by the electron density; field reversals are averaged out. This ambiguity can be solved, using polarization and RM studies of SNRs as anchor points, utilizing polarization surveys such as the Canadian Galactic Plane Survey (CGPS, Taylor et al. (2003), Landecker et al. (2010)).

The Model: I assume spherical, mature SNRs expanding into an environment of constant density and magnetic field. Emission and RM structure are computed as described in Kothes \& Brown (2008). The simulations show that ambient magnetic field directions can easily be determined from measurements of polarization angle corrected for Faraday rotation and the RM gradient on the bright SNR shells.

CTB104a: A CGPS radio polarization study of the SNR CTB104a (G93.7-0.2) indicated that the ambient magnetic field direction is opposite to the direction expected for this part of the Galaxy. A fit with the new model confirms this. The ambient magnetic field points towards us at an angle between $5-10^{\circ}$ with the plane of the sky and an ambient magnetic field strength of at least $10 \mu \mathrm{G}$.

G182.4+4.3: The SNR G182.4+4.3, discovered by Kothes, Fürst \& Reich (1998), is located close to the anti-centre of our Galaxy. If we assume that the magnetic field here is constant along the line of sight at about $4 \mu \mathrm{G}$, a fit to the SNRs RM structure reveals that we are looking perpendicular to the large-scale magnetic field at a Galactic longitude of $180^{\circ}$, which indicates an azimuthal magnetic field for the Outer Galaxy.

DA 530: The SNR DA $530($ G93.3+6.9) is located at high Galactic latitude above an area of the plane which is rich in SNRs, HII, and star formation regions. A model fit to this SNR's polarization and RM structure indicates that it is expanding into an area with twisted magnetic field lines. This is the kind of magnetic field structure that we would expect to find inside a Galactic chimney.

\section{References}

Kothes, R., Fürst, E., \& Reich, W. 1998, A\& $A 331,661$

Kothes, R. \& Brown, J.-A. 2008, Proc. IAU Symposium 259, 75

Landecker, T. L., Reich, W., Reid, R., et al. 2010, A\&GA 520, 80

Taylor, A. R., Gibson, S. J., Peracaula, M., et al. 2003, AJ 125, 3145

Uyanıker, B., Kothes, R., \& Brunt, C. M. 2002, ApJ 565, 1022 